\title{
STRICHARTZ ESTIMATES FOR THE SCHRÖDINGER EQUATION ON A TREE AND APPLICATIONS*
}

\author{
LIVIU I. IGNAT ${ }^{\dagger \ddagger}$
}

\begin{abstract}
In this paper we consider the Schrödinger equation on a network formed by a tree with the last generation of edges formed by infinite strips. We obtain Strichartz like estimates for the linear semigroup and apply them to obtain well-posedness results for a nonlinear problem.
\end{abstract}

Key words. Schrödinger equation, Strichartz estimates, quantum graphs

AMS subject classifications. 35R02,35Q55, 35B40

1. Introduction. Let us consider the linear Schrödinger equation (LSE):

$$
\left\{\begin{array}{l}
i u_{t}+u_{x x}=0, x \in \mathbb{R}, t \neq 0, \\
u(0, x)=\varphi(x), x \in \mathbb{R} .
\end{array}\right.
$$

The linear equation (1.1) is solved by $u(t, x)=S(t) \varphi$, where $S(t)=e^{i t \Delta}$ is the free Schrödinger operator. The linear semigroup has two important properties. First, the conservation of the $L^{2}$-norm:

$$
\|S(t) \varphi\|_{L^{2}(\mathbb{R})}=\|\varphi\|_{L^{2}(\mathbb{R})}
$$

and a dispersive estimate of the form:

$$
|(S(t) \varphi)(x)| \leq \frac{1}{(4 \pi|t|)^{1 / 2}}\|\varphi\|_{L^{1}(\mathbb{R})}, x \in \mathbb{R}, \quad t \neq 0 .
$$

The space-time estimate

$$
\|S(\cdot) \varphi\|_{L^{6}\left(\mathbb{R}, L^{6}(\mathbb{R})\right)} \leq C\|\varphi\|_{L^{2}(\mathbb{R})},
$$

due to Strichartz [16], is deeper. It guarantees that the solutions of system (1.1) decay as $t$ becomes large and that they gain some spatial integrability. Inequality (1.4) was generalized by Ginibre and Velo [9]. They proved the mixed space-time estimates, well known as Strichartz estimates:

$$
\|S(\cdot) \varphi\|_{L^{q}\left(\mathbb{R}, L^{r}(\mathbb{R})\right)} \leq C(q, r)\|\varphi\|_{L^{2}(\mathbb{R})}
$$

for the so-called admissible pairs $(q, r)$ :

$$
\frac{2}{q}+\frac{1}{r}=\frac{1}{2}, \quad 2 \leq q, r \leq \infty
$$

Similar results can be stated in any space dimension but it is beyond the scope of this article. These estimates have been successfully applied to obtain well-posedness results for the nonlinear Schrödinger equation (see [6], [17] and the reference therein).

\footnotetext{
*The author has been supported by the grants "Qualitative properties of the partial differential equations and their numerical approximations"-TE 4/2009 of CNCSIS Romania, LEA CNRS FrancoRoumain MATH-MODE, MTM2008-03541 of the Spanish MEC and the Project PI2010-04 of the Basque Government.

†Institute of Mathematics "Simion Stoilow" of the Romanian Academy, P.O. Box 1-764, RO014700 Bucharest, Romania. (liviu.ignat@gmail.com).

${ }^{\ddagger}$ BCAM Basque Center for Applied Mathematics, Bizkaia Technology Park, Building 500 Derio, Basque Country, Spain.
} 


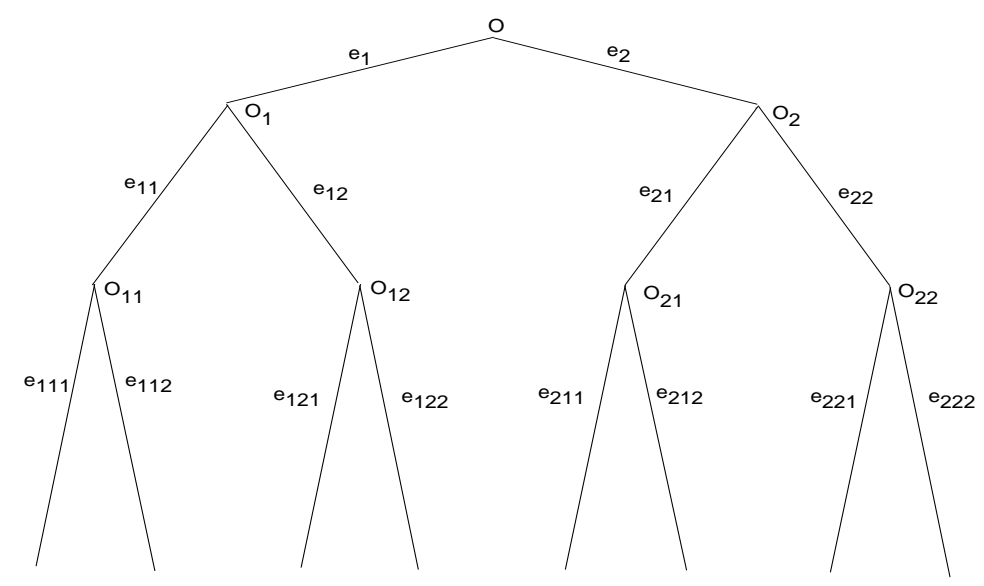

FIG. 1.1. A tree with the third generation formed by infinite edges

In this paper we consider the Schrödinger equation on a network formed by the edges of a tree, $\Gamma$, as in Fig. 1.1. We assume that each internal node of the tree has two children nodes, the edges at the same generation have the same length and the last generation of edges is formed by infinite strips. We prove that similar estimates to those mentioned above (1.5) hold in this case. The main idea behind our result is that solving the linear Schrödinger equation on such a structure could be reduced to solving an equation with finitely many piecewise constant coefficients on the whole real line.

The result obtained in this paper can be extended to regular trees $\Gamma$, i.e. trees having the property that all the vertices of the same generation have the same number of descendants and all the edges of the same generation are of the same length (see [15] for more details on regular trees), assuming that the last generation of edges is formed by infinite strips.

The problems we address here enter in the framework of quantum graphs. The name quantum graph is used for a graph considered as a one-dimensional singular variety and equipped with a differential operator. These quantum graphs arise as simplified models in mathematics, physics, chemistry, and engineering (e.g., nanotechnology and microelectronics), when one considers propagation of waves through a quasi-one-dimensional system that looks like a thin neighborhood of a graph. We can mention in particular the quantum wires and thin waveguides. We refer to the survey paper [11] and the references therein for more information on this topic.

The paper is organised as follows. In Section 2, for the sake of completeness, we introduce some notations, and basic facts about graphs and trees even if these are classical (see for example [7], [5]). We define the spaces $L^{2}(\Gamma), H^{m}(\Gamma)$ and introduce the Laplace operator on a graph network. Finally we write the Schrödinger equation on a graph network.

In Section 3 we analyse the dispersive properties of the LSE on the considered tree and apply them to the semilinear Schrödinger equation. In the last section we state some open problems.

2. Notations and Preliminaries. Let $\Gamma=(V, E)$ be a graph where $V$ is a set of vertices and $E$ the set of edges. For each $v \in V$ we denote $E_{v}=\{e \in E: v \in e\}$. 
We assume that $\Gamma$ is a countable connected locally finite graph, i.e. the degree of each vertex $v$ of $\Gamma$ is finite: $\left|E_{v}\right|<\infty$. The edges could be of finite length and then their ends are vertices of $V$ or they have infinite length and then we assume that each infinite edge is a ray with a single vertex belonging to $V$ (see [11] for more details on graphs with infinite edges).

We fix an orientation of $\Gamma$ and for each oriented edge $e$, we denote by $I(e)$ the initial vertex and by $T(e)$ the terminal one. Of course in the case of infinite edges we have only initial vertices.

We identify every edge $e$ of $\Gamma$ with an interval $I_{e}$, where $I_{e}=\left[0, l_{e}\right]$ if the edge is finite and $I_{e}=[0, \infty)$ if the edge is infinite. This identification introduces a coordinate $x_{e}$ along the edge $e$. In this way $\Gamma$ is a metric space and is often named metric graph $[11]$.

Let $v$ be a vertex of $V$ and $e$ be an edge in $E_{v}$. We set for finite edges $e$

$$
i(v, e)=\left\{\begin{array}{lll}
0 & \text { if } & v=I(e), \\
l_{e} & \text { if } & v=T(e)
\end{array}\right.
$$

and

$$
i(v, e)=0, \text { if } v=I(e)
$$

for infinite edges.

We identify any function $\mathbf{u}$ on $\Gamma$ with a collection $\left\{u^{e}\right\}_{e \in E}$ of functions $u^{e}$ defined on the edges $e$ of $\Gamma$. Each $u^{e}$ can be considered as a function on the interval $I_{e}$. In fact, we use the same notation $u^{e}$ for both the function on the edge $e$ and the function on the interval $I_{e}$ identified with $e$. For a function $\mathbf{u}: \Gamma \rightarrow \mathbb{C}, \mathbf{u}=\left\{u^{e}\right\}_{e \in E}$, we denote by $f(\mathbf{u}): \Gamma \rightarrow \mathbb{C}$ the family $\left\{f\left(u^{e}\right)\right\}_{e \in E}$, where $f\left(u^{e}\right): e \rightarrow \mathbb{C}$.

A function $\mathbf{u}=\left\{u^{e}\right\}_{e \in E}$ is continuous if and only if $u^{e}$ is continuous on $I_{e}$ for every $e \in E$, and moreover, it is continuous at the vertices of $\Gamma$ :

$$
u^{e}(i(v, e))=u^{e^{\prime}}\left(i\left(v, e^{\prime}\right)\right), \quad \forall e, e^{\prime} \in E_{v} .
$$

The space $L^{p}(\Gamma), 1 \leq p<\infty$ consists of all functions $\mathbf{u}=\left\{u_{e}\right\}_{e \in E}$ on $\Gamma$ that belong to $L^{p}\left(I_{e}\right)$ for each edge $e \in E$ and

$$
\|\mathbf{u}\|_{L^{p}(\Gamma)}^{p}=\sum_{e \in E}\left\|u^{e}\right\|_{L^{p}\left(I_{e}\right)}^{p}<\infty .
$$

Similarly, the space $L^{\infty}(\Gamma)$ consists of all functions that belong to $L^{\infty}\left(I_{e}\right)$ for each edge $e \in E$ and

$$
\|\mathbf{u}\|_{L^{\infty}(\Gamma)}=\sup _{e \in E}\left\|u^{e}\right\|_{L^{\infty}\left(I_{e}\right)}<\infty .
$$

The Sobolev space $H^{m}(\Gamma), m \geq 1$ an integer, consists in all continuous functions on $\Gamma$ that belong to $H^{m}\left(I_{e}\right)$ for each $e \in E$ and

$$
\|\mathbf{u}\|_{H^{m}(\Gamma)}^{2}=\sum_{e \in E}\left\|u^{e}\right\|_{H^{m}(e)}^{2}<\infty .
$$

The above spaces are Hilbert spaces with the inner products

$$
(\mathbf{u}, \mathbf{v})_{L^{2}(\Gamma)}=\sum_{e \in E}\left(u^{e}, v^{e}\right)_{L^{2}\left(I_{e}\right)}=\sum_{e \in E} \int_{I_{e}} u^{e}(x) \overline{v^{e}}(x) d x
$$


and

$$
(\mathbf{u}, \mathbf{v})_{H^{m}(\Gamma)}=\sum_{e \in E}\left(u^{e}, v^{e}\right)_{H^{m}\left(I_{e}\right)}=\sum_{e \in E} \sum_{k=0}^{m} \int_{I_{e}} \frac{d^{k} u^{e}}{d x^{k}} \frac{\overline{d^{k} v^{e}}}{d x^{k}} d x .
$$

We now introduce the Laplace operator $\Delta_{\Gamma}$ on the graph $\Gamma$. Even if it is a standard procedure we prefer for the sake of completeness to follow [4]. Consider the sesquilinear continuous form $\varphi$ on $H^{1}(\Gamma)$ defined by

$$
\varphi(\mathbf{u}, \mathbf{v})=\left(\mathbf{u}_{x}, \mathbf{v}_{x}\right)_{L^{2}(\Gamma)}=\sum_{e \in E} \int_{I_{e}} u_{x}^{e}(x) \overline{v_{x}^{e}}(x) d x .
$$

We denote by $D\left(\Delta_{\Gamma}\right)$ the set of all the functions $\mathbf{u} \in H^{1}(\Gamma)$ such that the linear map $\mathbf{v} \in H^{1}(\Gamma) \rightarrow \varphi_{\mathbf{u}}(\mathbf{v})=\varphi(\mathbf{u}, \mathbf{v})$ satisfies

$$
|\varphi(\mathbf{u}, \mathbf{v})| \leq C\|\mathbf{v}\|_{L^{2}(\Gamma)} \quad \text { for all } \mathbf{v} \in H^{1}(\Gamma) .
$$

For $\mathbf{u} \in D\left(\Delta_{\Gamma}\right)$, we can extend $\varphi_{\mathbf{u}}$ to an linear continuous mapping on $L^{2}(\Gamma)$. There is a unique element in $L^{2}(\Gamma)$ denoted by $\Delta_{\Gamma} \mathbf{u}$, such that,

$$
\varphi(\mathbf{u}, \mathbf{v})=-\left(\Delta_{\Gamma} \mathbf{u}, \mathbf{v}\right) \quad \text { for all } \mathbf{v} \in H^{1}(\Gamma) .
$$

We now define the normal exterior derivative of a function $\mathbf{u}=\left\{u^{e}\right\}_{e \in E}$ at the endpoints of the edges. For each $e \in E$ and $v$ an endpoint of $e$ we consider the normal derivative of the restriction of $\mathbf{u}$ to the edge $e$ of $E_{v}$ evaluated at $i(v, e)$ to be defined by:

$$
\frac{\partial u^{e}}{\partial n_{e}}(i(v, e))= \begin{cases}-u_{x}^{e}(0+) & \text { if } \quad i(v, e)=0, \\ u_{x}^{e}\left(l_{e}-\right) & \text { if } \quad i(v, e)=l_{e} .\end{cases}
$$

With this notation it is easy to characterise $D\left(\Delta_{\Gamma}\right)$ (see [4]):

$$
D\left(\Delta_{\Gamma}\right)=\left\{\mathbf{u}=\left\{u^{e}\right\}_{e \in E} \in H^{2}(\Gamma): \sum_{e \in E_{v}} \frac{\partial u^{e}}{\partial n_{e}}(i(v, e))=0 \quad \text { for all } v \in V\right\}
$$

and

$$
\left(\Delta_{\Gamma} \mathbf{u}\right)^{e}=\left(u^{e}\right)_{x x} \text { for all } e \in E, \mathbf{u} \in D\left(\Delta_{\Gamma}\right) .
$$

In other words $D\left(\Delta_{\Gamma}\right)$ is the space of all continuous functions on $\Gamma, \mathbf{u}=\left\{u^{e}\right\}_{e \in E}$, such that for every edge $e \in E, u^{e} \in H^{2}\left(I_{e}\right)$, and satisfying the following Kirchhoff-type condition:

$$
\sum_{e \in E: T(e)=v} u_{x}^{e}\left(l_{e}-\right)-\sum_{e \in E: I(e)=v} u_{x}^{e}(0+)=0 \quad \text { for all } v \in V .
$$

It is easy to verify that $\left(\Delta_{\Gamma}, D\left(\Delta_{\Gamma}\right)\right)$ is a linear, unbounded, self-adjoint, dissipative operator on $L^{2}(\Gamma)$, i.e. $\Re\left(\Delta_{\Gamma} \mathbf{u}, \mathbf{u}\right)_{L^{2}(\Gamma)} \leq 0$ for all $\mathbf{u} \in D\left(\Delta_{\Gamma}\right)$.

Let us consider the LSE on $\Gamma$ :

$$
\begin{cases}i \mathbf{u}_{t}(t, x)+\Delta_{\Gamma} \mathbf{u}(t, x)=0, & x \in \Gamma, t \neq 0 \\ \mathbf{u}(0)=\mathbf{u}_{0}, & x \in \Gamma .\end{cases}
$$


Using the properties of the operator $i \Delta_{\Gamma}$ we obtain as a consequence of the HilleYosida theorem the following well-posedness result.

THEOREM 2.1. For any $\mathbf{u}_{0} \in D\left(\Delta_{\Gamma}\right)$ there exists a unique solution $\mathbf{u}(t)$ of system (2.1) that satisfies

$$
\mathbf{u} \in C\left(\mathbb{R}, D\left(\Delta_{\Gamma}\right)\right) \cap C^{1}\left(\mathbb{R}, L^{2}(\Gamma)\right) .
$$

Moreover, for any $\mathbf{u}_{0} \in L^{2}(\Gamma)$, there exists a unique solution $\mathbf{u} \in C\left(\mathbb{R}, L^{2}(\Gamma)\right)$ that satisfies

$$
\|\mathbf{u}(t)\|_{L^{2}(\Gamma)}=\left\|\mathbf{u}_{0}\right\|_{L^{2}(\Gamma)} \quad \text { for all } t \in \mathbb{R}
$$

The $L^{2}(\Gamma)$-isometry property is a consequence of the fact that the operator $i \Delta_{\Gamma}$ satisfies $\Re\left(i \Delta_{\Gamma} \mathbf{u}, \mathbf{u}\right)_{L^{2}(\Gamma)}=0$ for all $\mathbf{u} \in D\left(\Delta_{\Gamma}\right)$.

3. Strichartz estimates for the Schrödinger equation on a tree. In what follows we consider a tree (planar, connected graph without closed paths) $\Gamma=(E, V)$. All the vertices, except the root $O$ that has multiplicity two, have multiplicity three, i.e. the number of edges that branch out from each vertex is three (see Fig. 1.1). Also the edges of the same generation have the same length (infinite in the case of the last generation of edges).

In this section we obtain Strichartz estimates for the solutions of the LSE on the network formed by the edges of the tree $\Gamma$. Following the same arguments we can extend the results presented here to the case of a regular tree.

Using the notations introduced in Section 2 we consider system (2.1) on the tree $\Gamma$ and denote its solution by $S_{\Delta_{\Gamma}}(t) \mathbf{u}_{0}=\exp \left(i t \Delta_{\Gamma}\right) \mathbf{u}_{0}$.

In the following theorem we state the dispersive property of the linear semigroup $S_{\Delta_{\Gamma}}(t)$.

TheOREM 3.1. For $p \in[2, \infty]$ and $t \neq 0, S_{\Delta_{\Gamma}}(t)$ maps $L^{p^{\prime}}(\Gamma)$ continuously to $L^{p}(\Gamma)$ and

$$
\left\|S_{\Delta_{\Gamma}}(t) \mathbf{u}_{0}\right\|_{L^{p}(\Gamma)} \leq C(\Gamma)|t|^{-\left(\frac{1}{2}-\frac{1}{p}\right)}\left\|\mathbf{u}_{0}\right\|_{L^{p^{\prime}}(\Gamma)}, \quad \text { for all } \mathbf{u}_{0} \in L^{p^{\prime}}(\Gamma) .
$$

The above result permits us to obtain more general estimates on the linear semigroup.

THEOREM 3.2. (Strichartz estimates) The linear semigroup $S_{\Delta_{\Gamma}}(t)$ satisfies the following properties

$$
\begin{gathered}
\left\|S_{\Delta_{\Gamma}}(t) \mathbf{u}_{0}\right\|_{L^{q}\left(\mathbb{R}, L^{r}(\Gamma)\right.} \leq C(\Gamma, q)\left\|\mathbf{u}_{0}\right\|_{L^{2}(\Gamma)}, \\
\left\|\int_{\mathbb{R}} S_{\Delta_{\Gamma}}(s)^{*} \mathbf{F}(s, \cdot) d s\right\|_{L^{2}(\Gamma)} \leq C(\Gamma, q)\|\mathbf{F}\|_{L^{q^{\prime}}\left(\mathbb{R}, L^{\left.r^{\prime}(\Gamma)\right)}\right.}, \\
\left\|\int_{0}^{t} S_{\Delta_{\Gamma}}(t-s) \mathbf{F}(s) d s\right\|_{L^{q}\left(\mathbb{R}, L^{r}(\Gamma)\right)} \leq C(\Gamma, q, \tilde{q})\|\mathbf{F}\|_{L^{\tilde{q}^{\prime}}\left(\mathbb{R}, L^{\tilde{r}^{\prime}}(\Gamma)\right)}
\end{gathered}
$$

for all admissible pairs $(q, r)$ and $(\tilde{q}, \tilde{r})$ satisfying (1.6). 
REMARK 3.3. All the constants in Theorem 3.1 and Theorem 3.2 depend on the number of the generations of the tree $\Gamma$ and not on the length of the edges. In the case of a regular tree with all the vertices at the generation $k$ having each one $d_{k+1}$ descendants, $0 \leq k \leq n$, the constants will also depend on the sequence $\left\{d_{k}\right\}_{k=1}^{n+1}$. In the considered case $d_{k}=2$ for all $1 \leq k \leq n+1$. At the end of the proof of Theorem 3.1 we will sketch how our argument can be adapted to the case of a regular tree.

As we have said in the introduction the Strichartz estimates have been used in the context of the nonlinear Schrödinger equation (NSE) to obtain well-posedness results. The arguments used in the context of NSE on the whole space can be used here since we have similar Strichartz like estimates on the linear semigroup generated by $i \Delta_{\Gamma}$, $S_{\Delta_{\Gamma}}(t)$.

THEOREM 3.4. Let $p \in(0,4)$. For any $\mathbf{u}_{0} \in L^{2}(\Gamma)$ there exists a unique solution

$$
\mathbf{u} \in C\left(\mathbb{R}, L^{2}(\Gamma)\right) \cap \bigcap_{(q, r) \text { admissible }} L_{\text {loc }}^{q}\left(\mathbb{R}, L^{r}(\Gamma)\right),
$$

of the equation

$$
\begin{cases}i \mathbf{u}_{t}+\Delta_{\Gamma} \mathbf{u} \pm|\mathbf{u}|^{p} \mathbf{u}=0, & t \neq 0 \\ \mathbf{u}(0)=\mathbf{u}_{0}, & t=0\end{cases}
$$

Moreover, the $L^{2}(\Gamma)$-norm of $\mathbf{u}$ is conserved along the time

$$
\|\mathbf{u}(t)\|_{L^{2}(\Gamma)}=\left\|\mathbf{u}_{0}\right\|_{L^{2}(\Gamma)} .
$$

The proof is standard once Theorem 3.2 is obtained and it follows the same lines as in [6], p. 109, Theorem 4.6.1. We leave it to the reader.

In order to proceed to the proof of Theorem 3.1 and Theorem 3.2 we now describe the procedure of indexing the edges and vertices of the tree (see Fig. 1.1). For each index $\bar{\alpha}=\left(\alpha_{1}, \alpha_{2}, \ldots, \alpha_{k}\right) \in\{1,2\}^{k}$ we denote by $|\bar{\alpha}|$ the number of its components: $|\bar{\alpha}|=k$.

The root of the tree is denoted by $O$. The remaining vertices and edges will be denoted by $O_{\bar{\alpha}},|\bar{\alpha}| \leq n$ and $e_{\bar{\alpha}},|\bar{\alpha}| \leq n+1$, respectively. Here $n$ and $n+1$ represent the number of generations of vertices, respectively of edges.

The vertices and edges are defined by recurrence in the following way. For each vertex $O_{\bar{\alpha}}$ with the index $|\bar{\alpha}| \leq n$ (possibly empty in the case of the root $O$ ) there are two edges that branch out from it $e_{\overline{\alpha \beta}}$ where $\overline{\alpha \beta}=\left(\alpha_{1}, \alpha_{2}, \ldots, \alpha_{k}, \beta\right)$ and $\beta \in\{1,2\}$. If $|\bar{\alpha}| \leq n-1$ the other endpoint of $e_{\overline{\alpha \beta}}$ will be denoted by $O_{\overline{\alpha \beta}}$. In the case when $|\bar{\alpha}|=n$ the edges that branch out from these vertices are infinite strips.

With our notations $E=\left\{e_{\bar{\alpha}}: \bar{\alpha} \in\{1,2\}^{k}, 1 \leq k \leq n+1\right\}$. A function $\mathbf{u}: \Gamma \rightarrow \mathbb{C}$ is a collection of functions $\left\{u_{\bar{\alpha}}\right\}_{e_{\bar{\alpha}} \in E}$ where each component $u_{\bar{\alpha}}$ is a function defined on the corresponding edge $e_{\bar{\alpha}}, u_{\bar{\alpha}}: e_{\bar{\alpha}} \rightarrow \mathbb{C}$. Each edge $e_{\bar{\alpha}}$ will be identified with the interval $\left[0, l_{|\bar{\alpha}|}\right]$ if $|\bar{\alpha}| \leq n$ and with $[0, \infty)$ if $|\bar{\alpha}|=n+1$.

Before starting the proof, since it is quite technical, let us point out its main steps. Equation (2.1) gives us a system of LSE on intervals of the type $\left(0, l_{k}\right)$ or $(0, \infty)$ coupled by Kirchhoff's law. Using a translation in the space variable we transform our problem to a system of coupled linear Schrödinger equations on some intervals $\left(a_{|\bar{\alpha}|-1}, a_{|\bar{\alpha}|}\right)$ or $\left(a_{n}, \infty\right)$ where the sequence $\left\{a_{k}\right\}_{k=0}^{n}$ depends on the length of the edges. We then define the functions $Z^{\bar{\alpha}}$ that equal the average of the functions 
defined on the edges emanating from the vertex $O_{|\alpha|}$ and its descendants. Using an inductive argument on $|\bar{\alpha}|$ we prove dispersive estimates for $Z^{\bar{\alpha}}$ which give us the same ones for $\mathbf{u}$.

Proof of Theorem 3.1. We recall that Theorem 2.1 shows that $S_{\Delta_{\Gamma}}(t)$ in an $L^{2}(\Gamma)$ isometry. Thus it is sufficient to prove that for any $t \neq 0, S_{\Delta_{\Gamma}}(t)$ maps continuously $L^{1}(\Gamma)$ to $L^{\infty}(\Gamma)$ with a norm less than $C(\Gamma)|t|^{-1 / 2}$. By density we can consider $\mathbf{u}_{0} \in D\left(\Delta_{\Gamma}\right)$ and prove the following estimate:

$$
\left\|S_{\Delta_{\Gamma}}(t) \mathbf{u}_{0}\right\|_{L^{\infty}(\Gamma)} \leq C(\Gamma)|t|^{-\frac{1}{2}}\left\|\mathbf{u}_{0}\right\|_{L^{1}(\Gamma)} \quad \text { for all } t \neq 0 .
$$
$C(n)$.

In the following we prove that the above estimate holds with a constant $C(\Gamma)=$

Using Theorem 2.1 in the particular case of the tree considered here we have that the solution of system (2.1) satisfies

$$
S_{\Delta_{\Gamma}}(t) \mathbf{u}_{0}=\left(u^{\bar{\alpha}}(t)\right)_{|\bar{\alpha}| \leq n+1} \in C\left(\mathbb{R}, D\left(\Delta_{\Gamma}\right)\right) \cap C^{1}\left(\mathbb{R}, L^{2}(\Gamma)\right) .
$$

It means that for all $t \in \mathbb{R}$ the functions $u^{\bar{\alpha}},|\bar{\alpha}| \leq n+1$, satisfy

$$
u^{\bar{\alpha}}(t) \in \begin{cases}H^{2}\left(0, l_{|\alpha|}\right), & |\bar{\alpha}| \leq n, \\ H^{2}(0, \infty), & |\bar{\alpha}|=n+1 .\end{cases}
$$

Moreover, the family $\left\{u^{\bar{\alpha}}\right\}_{1 \leq|\bar{\alpha}| \leq n+1}$ solves the following system:

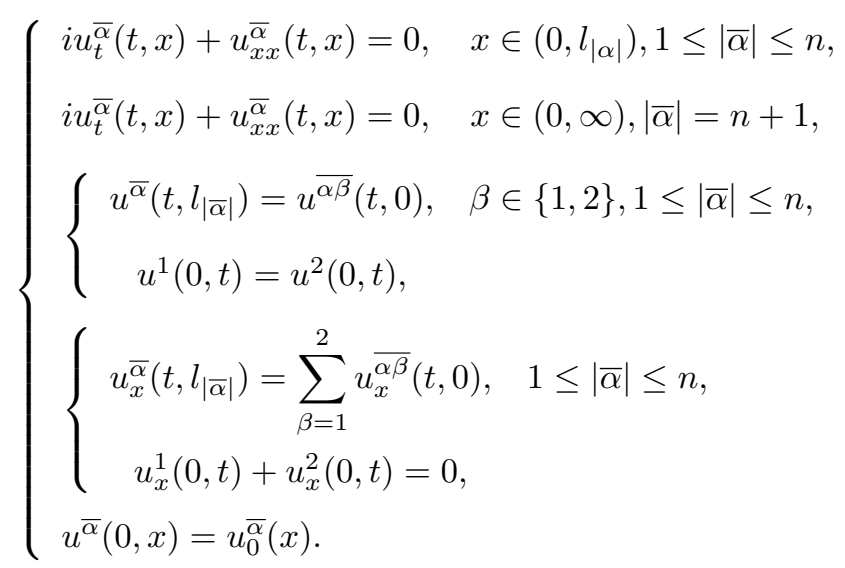

The first two equations represent the LSE satisfied by each $u^{\bar{\alpha}}$. The second type of properties gives the continuity at the ends of the vertices and the third one is the Kirchhoff type condition on the normal derivatives.

However, the above system is not very useful in order to reduce it to a LSE with discontinuous coefficients as we announced in the introduction. We will rewrite the above system in a convenient manner that will allow us to apply previous results on the dispersive properties of the Schrödinger equation $i u_{t}+\left(\sigma u_{x}\right)_{x}=0$ (see [1]), where $\sigma$ is a step function taking a finite number of values.

We consider the intervals

$$
I_{k}=\left\{\begin{array}{ccc}
\left(a_{k-1}, a_{k}\right) & \text { if } \quad 1 \leq k \leq n, \\
\left(a_{n}, \infty\right) & \text { if } \quad k=n+1
\end{array}\right.
$$




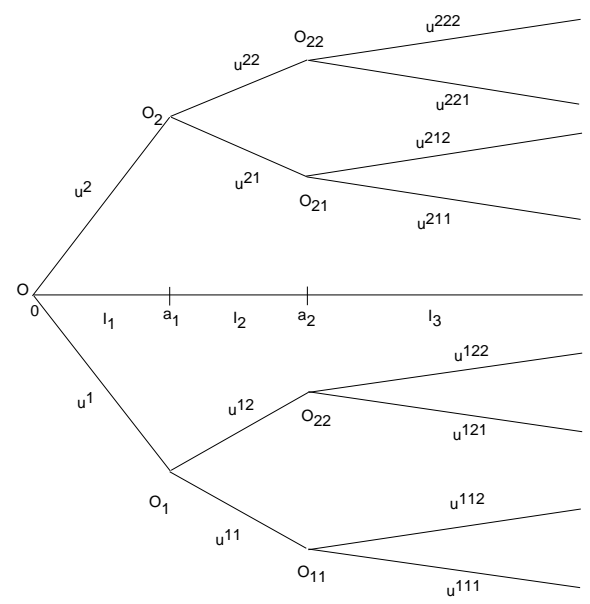

FIG. 3.1. The functions situated above each interval are defined on that interval, for example $u^{1}$ and $u^{2}$ are defined on $I_{1}$, etc.

where $a_{0}=0$ and $a_{k+1}=a_{k}+l_{k+1}$ for $k=0, \ldots, n-1$.

With the new notations, by applying translations in the space variable, system (3.6) can be written in an equivalent form:

$$
\left\{\begin{array}{l}
i u_{t}^{\bar{\alpha}}(t, x)+u_{x x}^{\bar{\alpha}}(t, x)=0, \quad x \in I_{|\overline{\mid}|}, 1 \leq|\bar{\alpha}| \leq n+1, \\
\left\{\begin{array}{l}
u^{\bar{\alpha}}\left(t, a_{|\bar{\alpha}|}\right)=u^{\overline{\alpha \beta}}\left(t, a_{|\bar{\alpha}|}\right), \quad \beta \in\{1,2\}, 1 \leq|\bar{\alpha}| \leq n, \\
u^{1}(t, 0)=u^{2}(t, 0),
\end{array}\right. \\
\left\{\begin{array}{l}
u_{x}^{\bar{\alpha}}\left(t, a_{|\bar{\alpha}|}\right)=\sum_{\beta=1}^{2} u_{x}^{\overline{\alpha \beta}}\left(t, a_{|\bar{\alpha}|}\right), \quad 1 \leq|\bar{\alpha}| \leq n, \\
u_{x}^{1}(t, 0)+u_{x}^{2}(t, 0)=0, \\
u^{\bar{\alpha}}(0, x)=u_{0}^{\bar{\alpha}}(x), \quad x \in I_{|\alpha|}, 1 \leq|\bar{\alpha}| \leq n .
\end{array}\right.
\end{array}\right.
$$

In Fig. 3.1 we can visualise where each function $u^{\bar{\alpha}}$ is defined after the translation. We point out that once the dispersive properties are obtained for the second system (3.7) they also hold for the first one (3.6). In the sequel we will concentrate on system (3.7) and prove that for each index $\bar{\alpha}$ with $|\bar{\alpha}|=k, 1 \leq k \leq n+1$, the following holds for all $t \neq 0$ :

$$
\max _{|\bar{\alpha}|=k}\left\|u^{\bar{\alpha}}(t)\right\|_{L^{\infty}\left(I_{k}\right)} \leq C(n)|t|^{-1 / 2} \sum_{1 \leq|\bar{\beta}| \leq n+1}\left\|u_{0}^{\bar{\beta}}\right\|_{L^{1}\left(I_{|\bar{\beta}|}\right)} .
$$

For any $\bar{\alpha}$ with $1 \leq|\bar{\alpha}| \leq n+1$ we define the functions

$$
Z^{\bar{\alpha}}: J_{|\bar{\alpha}|}=\bigcup_{k=0}^{n+1-|\bar{\alpha}|} I_{|\bar{\alpha}|+k} \rightarrow \mathbb{C}
$$




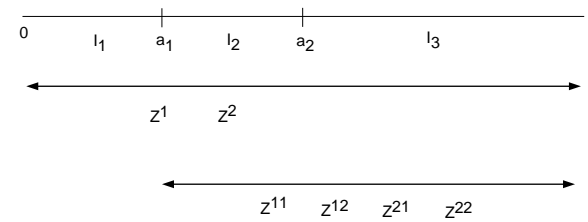

FIG. 3.2. The domain where the functions $Z^{\bar{\alpha}}$ are defined

as follows: for each $0 \leq k \leq n+1-|\alpha|$ and $x \in I_{|\alpha|+k}$ we set

$$
Z^{\bar{\alpha}}(t, x)=\frac{\sum_{|\bar{\beta}|=k} u^{\overline{\alpha \beta}}(t, x)}{2^{|\bar{\beta}|}} .
$$

The domain where each function $Z^{\bar{\alpha}}$ is defined can be viewed in Fig. 3.2.

The definition of the functions $Z^{\bar{\alpha}}$ shows that it coincides with $u^{\bar{\alpha}}$ on the interval $I_{|\bar{\alpha}|}$. Thus it is sufficient to prove that

$$
\left\|Z^{\bar{\alpha}}(t)\right\|_{L^{\infty}\left(J_{\bar{\alpha}}\right)} \leq c(n)|t|^{-1 / 2} \sum_{1 \leq|\bar{\beta}| \leq n+1}\left\|u_{0}^{\bar{\beta}}\right\|_{L^{1}\left(I_{\bar{\beta}}\right)} .
$$

In what follows it will be convenient to write $Z^{\bar{\alpha}}, 1 \leq|\bar{\alpha}| \leq n+1$, in a compact form:

$$
Z^{\bar{\alpha}}(t, x)=\sum_{k=0}^{n+1-|\bar{\alpha}|} \chi_{\left\{I_{|\bar{\alpha}|+k}\right\}}(x) \frac{1}{2^{k}} \sum_{|\bar{\beta}|=k} u^{\overline{\alpha \beta}}(t, x) .
$$

Moreover, the above identity shows that for each index $\bar{\alpha}$ with $1 \leq|\bar{\alpha}| \leq n+1$ we have the following inequality:

$$
\left\|Z^{\bar{\alpha}}(0)\right\|_{L^{1}\left(J_{|\bar{\alpha}|}\right)} \leq \sum_{|\bar{\alpha}| \leq|\bar{\beta}| \leq n+1}\left\|u_{0}^{\bar{\beta}}\right\|_{L^{1}\left(I_{|\bar{\beta}|}\right)} .
$$

To prove inequality (3.10) we need the following result that we will prove later.

Lemma 3.5. Let $n \geq 0,-\infty \leq a_{0}<a_{1}<\cdots<a_{n}<a_{n+1}=\infty$ and the function

$$
v \in C\left(\mathbb{R}, H^{2}\left(\left(a_{k}, a_{k+1}\right)\right)\right) \cap C^{1}\left(\mathbb{R}, L^{2}\left(\left(a_{k}, a_{k+1}\right)\right)\right), \quad k=0, \ldots, n
$$

which solves the following system

$$
\left\{\begin{array}{lc}
i v_{t}(t, x)+v_{x x}(t, x)=0, & x \in\left(a_{k}, a_{k+1}\right), 0 \leq k \leq n, t \neq 0, \\
v\left(t, a_{k}-\right)=v\left(t, a_{k}+\right), & 1 \leq k \leq n, \\
v_{x}\left(t, a_{k}-\right)=c_{k} v_{x}\left(t, a_{k}+\right), & t \neq 0,1 \leq k \leq n, \\
v\left(t, a_{0}\right)=0, & \text { if } a_{0}>-\infty, t \neq 0, \\
v(0, x)=v_{0}(x), & x \in\left(a_{k}, a_{k+1}\right), 0 \leq k \leq n,
\end{array}\right.
$$

for some positive constants $\left\{c_{k}\right\}_{k=1}^{n}$. 
If $v_{0} \in L^{1}\left(a_{0}, \infty\right)$ then there exists a positive constant $c\left(n,\left\{c_{k}\right\}_{k=1}^{n}\right)$ such that

$$
\|v(t)\|_{L^{\infty}\left(\left(a_{0}, \infty\right)\right)} \leq c\left(n,\left\{c_{k}\right\}_{k=1}^{n}\right)|t|^{-1 / 2}\left\|v_{0}\right\|_{L^{1}\left(\left(a_{0}, \infty\right)\right)} \quad \text { for all } t \neq 0 .
$$

Remark 3.6. The case $n=0, a_{0}=-\infty$, corresponds to system (1.1). The result in this case is classical [16].

REMARK 3.7. The constant in the right hand side of (3.14) does not depend on the sequence $\left\{a_{k}\right\}_{k=0}^{n+1}$.

We now prove inequality (3.10) by using an inductive argument following the length of the index $\bar{\alpha}$. We first consider the case when $|\bar{\alpha}|=1$.

Step 1. The first generation of $Z$ 's, $|\bar{\alpha}|=1$. We consider the functions

$$
Z(t, x)=\frac{Z^{1}(t, x)+Z^{2}(t, x)}{2}, \quad x \in(0, \infty), t \in \mathbb{R}
$$

and

$$
\tilde{Z}^{1}=Z-Z^{1}, \quad \tilde{Z}^{2}=Z-Z^{2} .
$$

We claim that

$$
\|Z(t)\|_{L^{\infty}((0, \infty))} \leq C(n)|t|^{-1 / 2}\left\|Z^{1}(0)+Z^{2}(0)\right\|_{L^{1}((0, \infty))}
$$

and

$$
\left\|\tilde{Z}^{1}(t)\right\|_{L^{\infty}((0, \infty))} \leq C(n)|t|^{-1 / 2}\left\|Z^{1}(0)-Z^{2}(0)\right\|_{L^{1}((0, \infty))} .
$$

A similar estimate will also hold for $\tilde{Z}^{2}(t)$.

In view of the definition of the functions $Z$ and $\tilde{Z}^{1}$ estimates (3.15) and (3.16) imply that

$$
\left\|Z^{1}(t)\right\|_{L^{\infty}((0, \infty))} \leq C(n)|t|^{-1 / 2}\left(\left\|Z^{1}(0)\right\|_{L^{1}((0, \infty))}+\left\|Z^{2}(0)\right\|_{L^{1}((0, \infty))}\right) .
$$

Thus, inequality (3.12) gives us that

$$
\left\|Z^{1}(t)\right\|_{L^{\infty}((0, \infty))} \leq C(n)|t|^{-1 / 2} \sum_{1 \leq|\bar{\beta}| \leq n+1}\left\|u_{0}^{\bar{\beta}}\right\|_{L^{1}\left(I_{|\bar{\beta}|}\right)},
$$

which proves estimate (3.10) in the considered case $|\bar{\alpha}|=1$.

We now prove estimates (3.15) and (3.16).

For the first one, we observe that $Z$ satisfies the system

$$
\left\{\begin{array}{cc}
i Z_{t}(t, x)+Z_{x x}(t, x)=0, & x \in \mathbb{R} \backslash\left\{a_{k}, 1 \leq k \leq n\right\}, \\
Z\left(t, a_{k}-\right)=Z\left(t, a_{k}+\right), & 1 \leq k \leq n, \\
Z_{x}\left(t, a_{k}-\right)=\frac{1}{2} Z_{x}\left(t, a_{k}+\right), & 1 \leq k \leq n, \\
Z_{x}(t, 0)=0, & t \in \mathbb{R}, \\
Z(0, x)=\frac{Z^{1}(0, x)+Z^{2}(0, x)}{2}, & x \in \mathbb{R} \backslash\left\{a_{k}, 1 \leq k \leq n\right\} .
\end{array}\right.
$$


Making an even extension of the function $Z$ to the whole real line we enter in the framework of Lemma 3.5 which gives us the following estimates for the function $Z$ :

$\|Z(t)\|_{L^{\infty}((0, \infty))} \leq C(n)|t|^{-1 / 2}\|Z(0)\|_{L^{1}((0, \infty))} \leq C(n)|t|^{-1 / 2}\left\|Z^{1}(0)+Z^{2}(0)\right\|_{L^{1}((0, \infty))}$.

This proves (3.15)

We now prove (3.16). The function $\tilde{Z}^{1}$ satisfies the following system:

$$
\left\{\begin{array}{cc}
i \tilde{Z}_{t}^{1}(t, x)+\tilde{Z}_{x x}^{1}(t, x)=0 & x \in \mathbb{R} \backslash\left\{a_{k}, 1 \leq k \leq n\right\}, \\
\tilde{Z}^{1}\left(t, a_{k}-\right)=\tilde{Z}^{1}\left(t, a_{k}+\right), & 1 \leq k \leq n, \\
\tilde{Z}^{1}(t, 0)=0, & t \in \mathbb{R}, \\
\tilde{Z}_{x}^{1}\left(t, a_{k}-\right)=\frac{1}{2} \tilde{Z}_{x}^{1}\left(t, a_{k}+\right), & 1 \leq k \leq n, \\
\tilde{Z}^{1}(0, x)=\frac{Z^{2}(0, x)-Z^{1}(0, x)}{2}, & x \in \mathbb{R} \backslash\left\{a_{k}, 1 \leq k \leq n\right\} .
\end{array}\right.
$$

We now apply Lemma 3.5 and we obtain that $\tilde{Z}^{1}$ satisfies

$\left\|\tilde{Z}^{1}(t)\right\|_{L^{\infty}((0, \infty))} \leq C(n)|t|^{-1 / 2}\left\|\tilde{Z}^{1}(0)\right\|_{L^{1}((0, \infty))} \leq C(n)|t|^{-1 / 2}\left\|Z^{1}(0)-Z^{2}(0)\right\|_{L^{1}((0, \infty))}$.

The proof of (3.10) in the case $|\bar{\alpha}|=1$ is now finished.

Step II. The next generations of $Z$ 's. We assume that we previously proved (3.10) for all indices $\bar{\alpha}$ with its length satisfying $|\bar{\alpha}|=k \leq n$. Let us choose an arbitrary index $\bar{\alpha}$ with $|\bar{\alpha}|=k$. We prove that (3.10) also holds for the index $\overline{\alpha \beta}$ with $\beta \in\{1,2\}$.

We consider the function

$$
\tilde{Z}^{\overline{\alpha \beta}}(t, x)=Z^{\overline{\alpha \beta}}(t, x)-Z^{\bar{\alpha}}(t, x), \quad x \in J_{k+1}=\bigcup_{m=k+1}^{n+1} I_{m} .
$$

In the case $k \leq n-1$ the new function $\tilde{Z}^{\overline{\alpha \beta}}$ satisfies the following system:

$$
\left\{\begin{array}{cc}
i \tilde{Z}_{t}^{\overline{\alpha \beta}}(t, x)+\tilde{Z}_{x x}^{\overline{\alpha \beta}}(t, x)=0, & t \neq 0, x \in \bigcup_{m=k+1}^{n+1} I_{m}, \\
\tilde{Z}^{\overline{\alpha \beta}}\left(t, a_{k}\right)=0, & t \neq 0, \\
\tilde{Z}^{\overline{\alpha \beta}}\left(t, a_{m}-\right)=\tilde{Z}^{\overline{\alpha \beta}}\left(t, a_{m}+\right), & k+1 \leq m \leq n, \\
\tilde{Z}_{x}^{\overline{\alpha \beta}}\left(t, a_{m}-\right)=\frac{1}{2} \tilde{Z}_{x}^{\overline{\alpha \beta}}\left(t, a_{m}+\right), & k+1 \leq m \leq n, \\
\tilde{Z}^{\alpha \beta}(0, x)=\tilde{Z}_{0}^{\overline{\alpha \beta}}(x), & x \in \bigcup_{m=k+1}^{n+1} I_{m} .
\end{array}\right.
$$

In the other case, $k=n$, we are dealing with an equation on the last generation of edges of the tree and we get the following system:

$$
\left\{\begin{array}{cc}
i \tilde{Z}_{t}^{\overline{\alpha \beta}}(t, x)+\tilde{Z}_{x x}^{\overline{\alpha \beta}}(t, x)=0, & t \neq 0, x \in I_{n+1}, \\
\tilde{Z}^{\overline{\alpha \beta}}\left(t, a_{n}\right)=0, & t \neq 0, \\
\tilde{Z}^{\overline{\alpha \beta}}(0, x)=\tilde{Z}_{0}^{\overline{\alpha \beta}}(x), & x \in I_{n+1} .
\end{array}\right.
$$


In both systems the second property is a consequence of the fact that $u^{\bar{\alpha}}\left(t, a_{k}\right)=$ $u^{\overline{\alpha \beta}}\left(t, a_{k}\right)$ and thus $Z^{\bar{\alpha}}\left(t, a_{k}\right)=Z^{\overline{\alpha \beta}}\left(t, a_{k}\right)$. The third and fourth properties in system (3.19) are given by the Kirchhoff type conditions imposed in system (3.7).

Systems (3.19) and (3.20) enter in the framework of Lemma 3.5. Thus, we have that

$$
\left\|\tilde{Z}^{\overline{\alpha \beta}}(t)\right\|_{L^{\infty}\left(J_{k+1}\right)} \leq C(n)|t|^{-1 / 2}\left\|\tilde{Z}_{0}^{\overline{\alpha \beta}}\right\|_{L^{1}\left(J_{k+1}\right)} .
$$

It follows that the same property holds for $Z^{\overline{\alpha \beta}}$ since $Z^{\overline{\alpha \beta}}=\tilde{Z}^{\overline{\alpha \beta}}+Z^{\bar{\alpha}}$. Indeed, for any $t \neq 0$ we have

$$
\begin{aligned}
\left\|Z^{\overline{\alpha \beta}}(t)\right\|_{L^{\infty}\left(J_{k+1}\right)} & \leq\left\|\tilde{Z}^{\overline{\alpha \beta}}(t)\right\|_{L^{\infty}\left(J_{k+1}\right)}+\left\|Z^{\bar{\alpha}}(t)\right\|_{L^{\infty}\left(J_{k+1}\right)} \\
& \leq\left\|\tilde{Z}^{\overline{\alpha \beta}}(t)\right\|_{L^{\infty}\left(J_{k+1}\right)}+\left\|Z^{\bar{\alpha}}(t)\right\|_{L^{\infty}\left(J_{k}\right)}
\end{aligned}
$$

and using the inductive assumption on $Z^{\bar{\alpha}}$ we obtain that

$$
\begin{aligned}
\left\|Z^{\overline{\alpha \beta}}(t)\right\|_{L^{\infty}\left(I_{k}\right)} & \leq C(n)|t|^{-1 / 2}\left(\left\|Z_{0}^{\overline{\alpha \beta}}-Z_{0}^{\bar{\alpha}}\right\|_{L^{1}\left(J_{k+1}\right)}+\left\|Z_{0}^{\bar{\alpha}}\right\|_{L^{1}\left(J_{k}\right)}\right) \\
& \leq C(n)|t|^{-1 / 2} \sum_{|\bar{\beta}| \leq n+1}\left\|u_{0}^{\bar{\beta}}\right\|_{L^{1}\left(I_{\bar{\beta}}\right)} .
\end{aligned}
$$

This implies that (3.10) holds for all indices with length $k+1$.

The proof of (3.10) is now finished.

Let us now comment about how the above proof can be adapted to obtain similar results in the case of a regular tree. Assume that all the vertices at the generation $k$ have $d_{k+1}$ descendants, $0 \leq k \leq n$, and all the edges of the same generation have the same length.

In this framework we have to modify the functions $Z^{\bar{\alpha}}$ in (3.9) in the following way:

$$
Z^{\bar{\alpha}}(t, x)= \begin{cases}u^{\bar{\alpha}} & x \in I_{|\bar{\alpha}|}, \\ \frac{\sum_{|\bar{\beta}|=k} u^{\overline{\alpha \beta}}(t, x)}{d_{|\bar{\alpha}|+1} \cdots d_{|\bar{\alpha}|+k}}, & x \in I_{|\bar{\alpha}|+k}, 1 \leq k \leq n+1-|\bar{\alpha}| .\end{cases}
$$

In Step I, we replace $Z$ by

$$
Z=\frac{Z^{1}+\cdots+Z^{d_{1}}}{d_{1}}, \tilde{Z}^{j}=Z-Z^{j}, j=1, \ldots, d_{1},
$$

the constant $1 / 2$ in coupling the derivatives at the points $a_{k}$ in systems (3.17) and (3.18) by $1 / d_{k+1}$ and the initial data in the two systems in agreement to the new definition of the functions $Z^{\bar{\alpha}}$. In Step II we replace in a similar manner the constant $1 / 2$ in systems (3.19) and (3.20) with $1 / d_{m+1}$ and the initial data.

The assumption on the geometry of the tree and the definition of the functions $Z^{\bar{\alpha}}$ as the average of the functions defined on the edges emanating from the vertex $O_{|\alpha|}$ and its descendants allow us to obtain the continuity property at the points $\left\{a_{k}\right\}_{k=1}^{n}$ in systems $(3.17-3.20)$.

With the above changes we can extend the results of Theorem 3.1, Theorem 3.2 and Theorem 3.4 to regular trees.

Before proceeding to prove Lemma 3.5 we recall the following result of Banica [1]. 
TheOREM 3.8. ([1]) Consider a partition of the real axis as follows:

$$
-\infty=x_{0}<x_{1}<\cdots<x_{n+1}=\infty
$$

and a step function

$$
\sigma(x)=\sigma_{i} \text { for } x \in\left(x_{i}, x_{i+1}\right),
$$

where $\sigma_{i}$ are positive numbers.

The solution $u$ of the Schrödinger equation

$$
\begin{cases}i u_{t}(t, x)+\left(\sigma(x) u_{x}\right)_{x}(t, x)=0, & \text { for } x \in \mathbb{R}, t \neq 0, \\ u(0, x)=u_{0}(x), & x \in \mathbb{R},\end{cases}
$$

satisfies the dispersion inequality

$$
\|u(t, \cdot)\|_{L^{\infty}(\mathbb{R})} \leq C|t|^{-1 / 2}\left\|u_{0}\right\|_{L^{1}(\mathbb{R})}, \quad t \neq 0,
$$

where the constant $C$ depends on $n$ and on the sequence $\left\{\sigma_{i}\right\}_{i=0}^{n}$.

Proof of Lemma 3.5. We first show how we can reduce the proof to the case $a_{0}=-\infty$. If $a_{0}>-\infty$ we can make a translation in the space variable and consider the case $a_{0}=0$. In this case we make an odd extension of the function $v$ and prove the desired properties for the extended function. The above argument allows us to consider only the case $a_{0}=-\infty$. Also the case $n=0$ is classical. Thus we will consider the case $n \geq 1$.

In the following we consider $a_{0}=-\infty$ and let $v$ be the solution of the following system

$$
\left\{\begin{array}{lc}
i v_{t}(t, x)+v_{x x}(t, x)=0, & x \in\left(a_{k}, a_{k+1}\right), 0 \leq k \leq n, t \neq 0, \\
v\left(t, a_{k}-\right)=v\left(t, a_{k}+\right), & 1 \leq k \leq n, \\
v_{x}\left(t, a_{k}-\right)=c_{k} v_{x}\left(t, a_{k}+\right), & 1 \leq k \leq n, \\
v(0, x)=v_{0}(x), & x \in\left(a_{k}, a_{k+1}\right), 0 \leq k \leq n .
\end{array}\right.
$$

For each $k=0, \ldots, n$ we define $T_{k}$ to be the linear function that maps the interval $\left(a_{k}, a_{k+1}\right)$ to $\left(b_{k}, b_{k+1}\right)$ where $\left\{b_{k}\right\}_{k=0}^{n}$ is an increasing sequence which will be defined later. More precisely $T_{k}$ satisfies:

$$
\left\{\begin{array}{lc}
T_{k}:\left(a_{k}, a_{k+1}\right) \rightarrow\left(b_{k}, b_{k+1}\right), & k=0, \ldots, n, \\
T_{k}\left(a_{k}\right)=b_{k}, T_{k}\left(a_{k+1}\right)=b_{k+1}, & k=0, \ldots, n \\
\left(T_{k}\right)_{x}(x)=\mu_{k}, & x \in\left(a_{k}, a_{k+1}\right), k=0, \ldots, n .
\end{array}\right.
$$

The constants $\left\{\mu_{k}\right\}_{k=0}^{n}$ will be chosen later in order to satisfy some compatibility conditions. We choose $b_{0}=a_{0}$ and $b_{n+1}=a_{n+1}$. The other constants $b_{k}, 1 \leq k \leq n$, are uniquely determined by the choice of the sequence $\left\{\mu_{k}\right\}_{k=1}^{n}$.

We introduce a new function $w$ depending on $v$ and show that it solves a LSE with a finite number of positive piecewise constant coefficients. Set

$$
w\left(T_{k}(x)\right)=v(x), \quad x \in\left(a_{k}, a_{k+1}\right), 0 \leq k \leq n .
$$


We claim that $w$ satisfies the following system

$$
\left\{\begin{array}{lc}
i w_{t}(t, x)+\mu_{k}^{2} w_{x x}(t, x)=0, & x \in\left(b_{k}, b_{k+1}\right), 0 \leq k \leq n, \\
w\left(t, b_{k}-\right)=w\left(t, b_{k}+\right), & 1 \leq k \leq n, \\
w_{x}\left(t, b_{k}-\right) \mu_{k-1}=c_{k} w_{x}\left(t, b_{k}+\right) \mu_{k}, & 1 \leq k \leq n, \\
w(x, 0)=w_{0}(x), & x \in\left(b_{k}, b_{k+1}\right), 0 \leq k \leq n .
\end{array}\right.
$$

We observe that for any $x \in\left(a_{k}, a_{k+1}\right), 0 \leq k \leq n$, we have

$$
i v_{t}(t, x)+v_{x x}(t, x)=i w_{t}\left(t, T_{k}(x)\right)+\mu_{k}^{2} w_{x x}\left(t, T_{k}(x)\right)=0, x \in\left(a_{k}, a_{k+1}\right) .
$$

This proves the first property in system (3.25). The second property holds since $v$ is continuous at each point $a_{k}$ with $1 \leq k \leq n$. For the third property we use that for each $1 \leq k \leq n$ the following hold

$$
v_{x}\left(a_{k}-\right)=w_{x}\left(b_{k}-\right) \mu_{k-1}
$$

and

$$
v_{x}\left(a_{k}+\right)=w_{x}\left(b_{k}+\right) \mu_{k} .
$$

We now choose the sequence $\left\{\mu_{k}\right\}_{k=0}^{n}$ such that the function $w$ defined in (3.24) satisfies the following equation on the whole real line

$$
\left\{\begin{array}{lc}
i w_{t}+\left(\sigma(x) w_{x}\right)_{x}=0, & x \in \mathbb{R}, t \neq 0, \\
w(0, x)=w_{0}(x), & x \in \mathbb{R},
\end{array}\right.
$$

where

$$
\sigma(x)=\mu_{k}^{2}, \quad x \in\left(b_{k}, b_{k+1}\right), \quad 0 \leq k \leq n .
$$

Observe that for (3.26) to hold with $\sigma$ defined by (3.27) we have to impose that

$$
\sigma\left(b_{k}+\right) w_{x}\left(t, b_{k}+\right)=\sigma\left(b_{k}-\right) w_{x}\left(t, b_{k}-\right), \quad k=1, \ldots, n .
$$

It means that

$$
\mu_{k}^{2} w_{x}\left(t, b_{k}+\right)=\mu_{k-1}^{2} w_{x}\left(t, b_{k}-\right), \quad k=1, \ldots, n .
$$

The third property in (3.25) requires that

$$
\mu_{k}=c_{k} \mu_{k-1} \quad \text { for } \quad k=1, \ldots, n .
$$

Choosing $\mu_{0}=1$ and $\mu_{k}=c_{1} \ldots c_{k}, k \geq 1$, the above property is satisfied and $w$ solves system (3.26) with $\sigma$ defined by (3.27).

We are now able to obtain the $L^{1}(\mathbb{R})-L^{\infty}(\mathbb{R})$ property for $v$ and $w$. Using Theorem 3.8 we obtain that $w$ satisfies

$$
\|w(t)\|_{L^{\infty}(\mathbb{R})} \leq c(\sigma)|t|^{-1 / 2}\left\|w_{0}\right\|_{L^{1}(\mathbb{R})},
$$


where $c(\sigma)=c\left(\left\{n,\left\{\mu_{k}\right\}_{k=0}^{n}\right\}\right)=c\left(\left\{n,\left\{c_{k}\right\}_{k=0}^{n}\right\}\right)$.

It is then obvious that $v$ satisfies property (3.14).

The proof is now finished.

Using Theorem 3.1 we can prove Theorem 3.2. To proceed we need the following result of Keel and Tao [10].

Proposition 3.9. ([10], Theorem 1.2) Let $H$ be a Hilbert space, $(X, d x)$ be a measure space and $U(t): H \rightarrow L^{2}(X)$ be a one parameter family of mappings with $t \in \mathbb{R}$, which obey the energy estimate

$$
\|U(t) f\|_{L^{2}(X)} \leq C\|f\|_{H}
$$

and the decay estimate

$$
\left\|U(t) U(s)^{*} g\right\|_{L^{\infty}(X)} \leq C|t-s|^{-1 / 2}\|g\|_{L^{1}(X)}
$$

Then

$$
\begin{gathered}
\|U(t) f\|_{L^{q}\left(\mathbb{R}, L^{r}(X)\right)} \leq C\|f\|_{H}, \\
\left.\| \int_{\mathbb{R}}(U(s))^{*} F(s, \cdot)\right) d s\left\|_{H} \leq C\right\| F \|_{L^{q^{\prime}}\left(\mathbb{R}, L^{r^{\prime}}(X)\right)}, \\
\left\|\int_{0}^{t} U(t-s) F(s) d s\right\|_{L^{q}\left(\mathbb{R}, L^{r}(X)\right)} \leq C\|F\|_{L^{\tilde{q}^{\prime}}\left(\mathbb{R}, L^{\tilde{r}^{\prime}}(X)\right)}
\end{gathered}
$$

for all admissible pairs $(q, r)$ and $(\tilde{q}, \tilde{r})$ satisfying (1.6).

Proof of Theorem 3.2. We apply Proposition 3.9 to the operators $\left\{S_{\Delta_{\Gamma}}(t)\right\}_{t \in \mathbb{R}}$ where $X=\Gamma$ and $H=L^{2}(\Gamma)$. The first hypothesis (3.29) is satisfied since $S_{\Delta_{\Gamma}}(t)$ is an $L^{2}(\Gamma)$-isometry.

In order to check the second hypothesis we point out that since $i \Delta_{\Gamma}$ is skew-adjoint the operator $S_{\Delta_{\Gamma}}(t)$ satisfies

$$
S_{\Delta_{\Gamma}}(t)^{*}=S_{\Delta_{\Gamma}}(-t)
$$

Thus by Theorem 3.1 we get

$$
\left\|S_{\Delta_{\Gamma}}(t) S_{\Delta_{\Gamma}}(s)^{*} g\right\|_{L^{\infty}(\Gamma)}=\left\|S_{\Delta_{\Gamma}}(t-s) g\right\|_{L^{\infty}(\Gamma)} \leq C(\Gamma)|t-s|^{-1 / 2}\|g\|_{L^{1}(\Gamma)}
$$

and hence hypothesis (3.30) is satisfied.

Applying Proposition 3.9 we obtain the desired result.

4. Some open problems. In this paper we have considered the case of a tree such that each internal vertex has two descendants and the results can be extended to the case of a regular tree as it was defined in [15]. Our proof does not cover the case when the edges of the tree have arbitrary lengths. The fact that all the edges at the same generation have the same length allowed us to consider the averages of the functions $u^{\bar{\alpha}}$ s defined at the same generation of edges by introducing the functions $Z^{\bar{\alpha}}$ in the proof of Theorem 3.1. In the case when the edges of a generation have different lengths we cannot define the functions $Z^{\bar{\alpha}}$ and our argument cannot be applied. This 


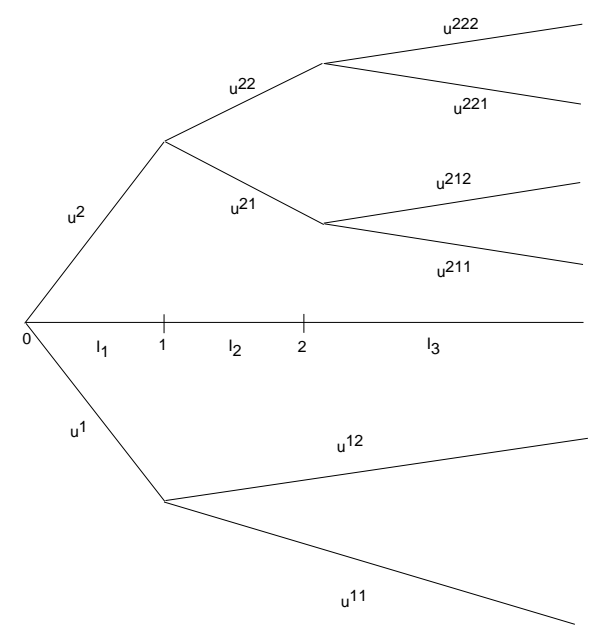

FIG. 3.3. A tree where the infinite strips occur at different levels

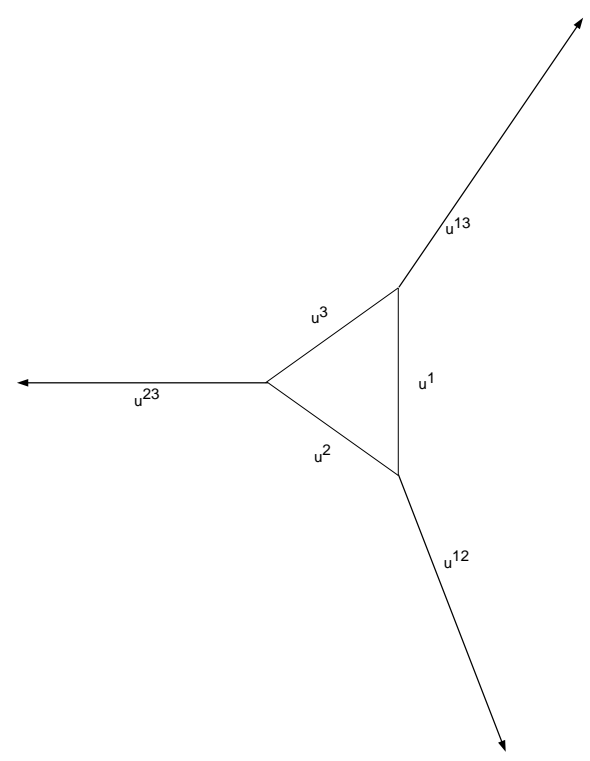

FIG. 3.4. A tree where two kinds of structures occur: a periodic one given by the triangle and the infinite strips

is the case, for example, of the tree in Fig. 3.3 where the functions $u^{\bar{\alpha}}, \bar{\alpha} \in\{1,2\}^{2}$ are not defined on the same interval.

On the other hand the proof of Theorem 3.1 cannot be applied to the case when at some generation we have two vertices with different number of descendants. The fact that at the same generation the number of descendants is the same help us obtain the continuity property at the points $\left\{a_{k}\right\}_{k=1}^{n}$ of the functions involved in systems (3.17 - 3.20) and then to use Lemma 3.5.

We conjecture that the results we obtained in the present paper can be extended to trees with the last generation of edges formed by infinite strips. In this context new 
methods have to be developed. We mention the works $[12,8,13,14]$ in the context of the asymptotic behavior of the heat kernels on trees and graphs networks.

Another interesting problem consists in the analysis of the same properties on some graphs which combine the periodic structure with the infinite strips. This is the case in Fig. 3.4. We recall that for LSE on the one-dimensional torus Bourgain [2] has analyzed the existence of Strichartz estimates. In the same framework we also mention the work of Burq et. al. [3].

Acknowledgements. The author thanks Enrique Zuazua for proposing this problem when both visited the Isaac Newton Institute, Cambridge, within the program "Highly Oscillatory Problems" and to Pavel Exner for pointing out the possible extension of the results to regular trees. The author also acknowledges the above institution and Professor A. Iserles for their hospitality and support.

Parts of this paper have been developed during the author's visit to BCAM Basque Center for Applied Mathematics under the Visiting Fellow program.

\section{REFERENCES}

[1] V. Banica. Dispersion and Strichartz inequalities for Schrödinger equations with singular coefficients. SIAM J. Math. Anal., 35(4):868-883 (electronic), 2003.

[2] J. Bourgain. Fourier transform restriction phenomena for certain lattice subsets and applications to nonlinear evolution equations. I. Schrödinger equations. Geom. Funct. Anal., 3(2):107-156, 1993.

[3] N. Burq, P. Gérard, and N. Tzvetkov. Strichartz inequalities and the nonlinear Schrödinger equation on compact manifolds. Amer. J. Math., 126(3):569-605, 2004.

[4] C. Cattaneo. The spectrum of the continuous Laplacian on a graph. Monatsh. Math., 124(3):215-235, 1997.

[5] C. Cattaneo and L. Fontana. D'Alembert formula on finite one-dimensional networks. J. Math. Anal. Appl., 284(2):403-424, 2003.

[6] T. Cazenave. Semilinear Schrödinger equations. Courant Lecture Notes in Mathematics 10. Providence, RI: American Mathematical Society (AMS); New York, NY: Courant Institute of Mathematical Sciences. xiii , 2003.

[7] R. Dáger and E. Zuazua. Wave propagation, observation and control in 1-d flexible multistructures, volume 50 of Mathématiques 8 Applications (Berlin) [Mathematics 83 Applications]. Springer-Verlag, Berlin, 2006.

[8] Bernard Gaveau, Masami Okada, and Tatsuya Okada. Explicit heat kernels on graphs and spectral analysis. In Several complex variables (Stockholm, 1987/1988), volume 38 of Math. Notes, pages 364-388. Princeton Univ. Press, Princeton, NJ, 1993.

[9] J. Ginibre and G. Velo. The global Cauchy problem for the nonlinear Schrödinger equation revisited. Ann. Inst. H. Poincaré Anal. Non Linéaire, 2(4):309-327, 1985.

[10] M. Keel and T. Tao. Endpoint Strichartz estimates. Am. J. Math., 120(5):955-980, 1998.

[11] P. Kuchment. Quantum graphs: an introduction and a brief survey. In Analysis on graphs and its applications, volume 77 of Proc. Sympos. Pure Math., pages 291-312. Amer. Math. Soc., Providence, RI, 2008.

[12] Masami Okada. Green function on self-similar trees. In Progress in differential geometry, volume 22 of Adv. Stud. Pure Math., pages 231-242. Math. Soc. Japan, Tokyo, 1993.

[13] Masami Okada, Takeshi Sekiguchi, and Yasunobu Shiota. Heat kernels on infinite graph networks and deformed Sierpiński gaskets. Japan J. Appl. Math., 7(3):527-543, 1990.

[14] Tatsuya Okada. Asymptotic behavior of skew conditional heat kernels on graph networks. Canad. J. Math., 45(4):863-878, 1993.

[15] Michael Solomyak. Laplace and Schrödinger operators on regular metric trees: the discrete spectrum case. In Function spaces, differential operators and nonlinear analysis (Teistungen, 2001), pages 161-181. Birkhäuser, Basel, 2003.

[16] R.S. Strichartz. Restrictions of Fourier transforms to quadratic surfaces and decay of solutions of wave equations. Duke Math. J., 44:705-714, 1977.

[17] T. Tao. Nonlinear dispersive equations, volume 106 of CBMS Regional Conference Series in Mathematics. Published for the Conference Board of the Mathematical Sciences, Washington, DC, 2006. Local and global analysis. 